\title{
On a nonlocal implicit problem under Atangana-Baleanu-Caputo fractional derivative
}

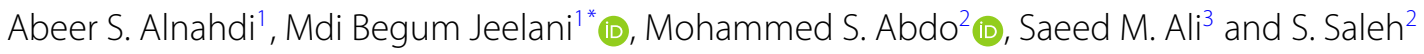

\section{"Correspondence:}

mbshaikh@imamu.edu.sa

${ }^{1}$ Department of Mathematics and

Statistics, Imam Mohammad Ibn

Saud Islamic University, Riyadh,

Saudi Arabia

Full list of author information is

available at the end of the article

\begin{abstract}
In this paper, we study a class of initial value problems for a nonlinear implicit fractional differential equation with nonlocal conditions involving the Atangana-Baleanu-Caputo fractional derivative. The applied fractional operator is based on a nonsingular and nonlocal kernel. Then we derive a formula for the solution through the equivalent fractional functional integral equations to the proposed problem. The existence and uniqueness are obtained by means of Schauder's and Banach's fixed point theorems. Moreover, two types of the continuous dependence of solutions to such equations are discussed. Finally, the paper includes two examples to substantiate the validity of the main results.
\end{abstract}

MSC: 26A33; 34A08; 34A12; 34B15

Keywords: ABC-type fractional differential equation; Nonlocal conditions; Fixed point theorem

\section{Introduction}

Fractional calculus $[1,2]$ has persistently magnetized the attention of many researchers in the few past decades. Recently, novel fractional derivatives which mix the RiemannLiouville, Caputo, Hadamard, Hilfer, and generalized fractional derivatives have emerged (see [3-8]). Some interested authors and researchers have realized that innovation for novel fractional derivatives with nonsingular (nonlocal) or singular (local) kernels is an urgent necessity to satisfy the need to model more realistic problems in different fields of applied science.

Caputo and Fabrizio in [9] suggested a novel kind of fractional derivatives where the kernel relies on the exponential function. Some properties of this novel operator were studied by Losada and Nieto in [10]. In [11] the authors proposed interesting new fractional operators called Atangana-Baleanu (AB) fractional operators. One of these operators is called Atangana-Baleanu-Caputo $(\mathrm{ABC})$ fractional derivative, and it is basically a generalization of the Caputo operator. Then, in $[12,13]$, the authors discussed the discrete versions of those novel operators. Some recent and interesting contributions on fractional differential

(c) The Author(s) 2021. This article is licensed under a Creative Commons Attribution 4.0 International License, which permits use sharing, adaptation, distribution and reproduction in any medium or format, as long as you give appropriate credit to the original author(s) and the source, provide a link to the Creative Commons licence, and indicate if changes were made. The images or other third party material in this article are included in the article's Creative Commons licence, unless indicated otherwise in a credit line to the material. If material is not included in the article's Creative Commons licence and your intended use is not permitted by statutory regulation or exceeds the permitted use, you will need to obtain permission directly from the copyright holder. To view a copy of this licence, visit http://creativecommons.org/licenses/by/4.0/. 
equations (FDEs) and mathematical modeling that incorporate $\mathrm{ABC}$ fractional derivatives can be found in the following series of articles [14-26].

The recent investigations of the qualitative analysis of FDEs, e.g., the evolution, impulsive, and functional problems with initial (or boundary) nonlocal conditions, can be found in [27-35] and the references therein.

On the other hand, in the case where a physical procedure is described by IVPs for FDEs, at that point it is desirable that any mistakes made in the estimation of initial data do not impact the solution so much. Mathematically, this is known as continuous dependence of solution of an IVP on the data introduced in the proposed problem. Actually, nonlocal conditions come up when estimations of the function on the limit are associated with values in the domain. It is seen as more reasonable than the classical initial conditions for the forming of some physical phenomena in specific problems of wave spread and thermodynamics. In crossing, we saw that the nonlocal condition $\sum_{k=1}^{m} \beta_{k} \varkappa_{(}\left(\tau_{k}\right)=\varkappa_{0}$ that may be applied in physical models yields preferred impact over the initial conditions $\varkappa(0)=\varkappa_{0}$.

In this regard, many interested authors have presented excellent results on the existence and continuous dependence of solution of FDEs with the nonlocal conditions and classical fractional operators. For the recent review of these studies, we refer to [36-44].

Recently, ABC-fractional IVP is one of the studied problems by Thabet et al. [14] which is of type

$$
\left\{\begin{array}{l}
A B C \mathbb{D}_{a, \theta}^{\varrho} \varkappa(\theta)=f(\theta, \varkappa(\theta)), \quad \theta \in[a, \chi], 0<\varrho \leq 1, \\
\varkappa(a)=\varkappa_{0} .
\end{array}\right.
$$

Through the above discussions, and motivated by [14, 40], in this work, we will prove some new results based on a novel version of fractional operators. More precisely, we consider the following $\mathrm{ABC}$-type nonlocal fractional problem:

$$
\begin{aligned}
& A B \mathbb{D}_{0, \theta}^{\varrho} \varkappa(\theta)=f\left(\theta, \varkappa(\theta),{ }^{A B C} \mathbb{D}_{0, \theta}^{\varrho} \varkappa(\theta)\right), \quad \theta \in[0, \chi], \\
& \sum_{k=1}^{m} \beta_{k} \varkappa\left(\tau_{k}\right)=\varkappa_{0}, \quad \tau_{k} \in(0, \chi),
\end{aligned}
$$

where $0<\varrho \leq 1,{ }^{A B C} \mathbb{D}_{a^{+}}^{\varrho}$ is the $\mathrm{ABC}$ fractional derivative of order $\varrho, f:[0, \chi] \times \mathbb{R} \times \mathbb{R} \rightarrow \mathbb{R}$ is a continuous function with $f\left(0, \varkappa(0),{ }^{A B C} \mathbb{D}_{0, \theta}^{\varrho} \varkappa(0)\right)=0,0<\tau_{1}<\tau_{2}<\cdots<\tau_{m}<\chi, \beta_{k}$ are real numbers $(k=1,2, \ldots, m)$, and $\varkappa \in C[0, \chi]$ such that the operator ${ }^{A B C} \mathbb{D}_{0, \theta}^{\varrho}$ exists and $A B C \mathbb{D}_{0, \theta}^{\varrho} \varkappa \in C[0, \chi]$.

The main aim of this work is to study the existence, uniqueness of solutions and their continuous dependence on the nonlinear nonlocal problem (1.1)-(1.2) in the frame of $\mathrm{ABC}$ fractional derivative by means of Schauder's and Banach's fixed point theorems. To the best of our knowledge in the subject, no one considered the existence and data dependence of the $\mathrm{ABC}$-type fractional problem with nonlocal conditions. Therefore, the acquired results are recent studies and an extension of the development of FDEs involving an $\mathrm{ABC}$ fractional derivative. Furthermore, the analysis of the results is restricted to a minimum of hypotheses.

The rest of the paper is arranged as follows. In Sect. 2, we recall some useful preliminaries related to the main outcomes. Section 3 is dedicated to obtaining the solution representation to a given problem. Then the existence and uniqueness results are proved via 
functional integral equation with the aid of some fixed point approaches. Moreover, we discuss the continuous dependence of solutions for the problem at hand. Illustrative examples are given in Sect. 4. Finally, concluding remarks are mentioned in Sect. 5.

\section{Background materials and preliminaries}

Here, we recall some essential definitions and preliminary facts related to AB fractional operators.

Let $C([0, \chi], \mathbb{R})=C[0, \chi]$ be the space of continuous functions $v:[0, \chi] \rightarrow \mathbb{R}$ with the norm

$$
\|v\|=\max \{|v(\theta)|: \theta \in[0, \chi]\} .
$$

Clearly, $C[0, \chi]$ is a Banach space with the norm $\|\cdot\|$.

Definition 2.1 ([11]) Let $\varrho \in[0,1]$ and $v \in H^{1}(0, \chi)$. Then the AB-Riemann-Liouville and $A B-C a p u t o$ fractional derivatives are given by

$$
{ }_{A B R} \mathbb{D}_{a^{+}, \theta}^{\varrho} v(\theta)=\frac{\mathfrak{N}(\varrho)}{1-\varrho} \frac{d}{d \theta} \int_{a}^{\theta} \mathbb{E}_{\varrho}\left(\frac{-\varrho}{1-\varrho}(\theta-\sigma)^{\varrho}\right) v(\sigma) d \sigma, \quad \theta>a,
$$

and

$$
{ }^{A B C} \mathbb{D}_{a^{+}, \theta}^{\varrho} v(\theta)=\frac{\mathfrak{N}(\varrho)}{1-\varrho} \int_{a}^{\theta} \mathbb{E}_{\varrho}\left(\frac{-\varrho}{1-\varrho}(\theta-\sigma)^{\varrho}\right) v^{\prime}(\sigma) d \sigma, \quad \theta>a
$$

respectively, where $\mathbb{E}_{\varrho}$ is called the MLF defined by

$$
\mathbb{E}_{\varrho}(\theta)=\sum_{k=0}^{\infty} \frac{\theta^{k}}{\Gamma(k \varrho+1)}, \quad \operatorname{Re}(\varrho)>0, \quad \theta \in \mathbb{C} .
$$

The $\mathrm{AB}$ fractional integral is described by

$$
{ }^{A B} \mathbb{I}_{a^{+}, \theta}^{\varrho} v(\theta)=\frac{1-\varrho}{\mathfrak{N}(\varrho)} v(\theta)+\frac{\varrho}{\mathfrak{N}(\varrho)} I_{a^{+}}^{\varrho} v(\theta), \quad \theta>a,
$$

where $\mathfrak{N}(\varrho)>0$ is a normalization function satisfying $\mathfrak{N}(0)=\mathfrak{N}(1)=1$ and

$$
I_{a^{+}}^{\varrho} v(\theta)=\frac{1}{\Gamma(\varrho)} \int_{a}^{\theta}(\theta-\sigma)^{\varrho-1} v(\sigma) d \sigma .
$$

Lemma 2.2 $([11,45])$ Let $\varrho \in(0,1]$ and $v \in H^{1}(0, \chi)$, if an $A B C$ fractional derivative exists, then we have

$$
{ }^{A B C} \mathbb{D}_{a^{+}, \theta}^{\varrho} A B \mathbb{I}_{a^{+}, \theta}^{\varrho} v(\theta)=v(\theta)
$$

and

$$
A B \mathbb{I}_{a^{+}, \theta}^{\varrho} A B C \mathbb{D}_{a^{+}, \theta}^{\varrho} v(\theta)=v(\theta)-v(a) .
$$


Definition 2.3 ([11]) The relation between the AB-Caputo and AB-Riemann-Liouville operator is

$$
{ }_{A B C} \mathbb{D}_{a^{+}, \theta}^{\varrho} v(\theta)={ }^{A B R} \mathbb{D}_{a^{+}, \theta}^{\varrho} v(\theta)-\frac{\mathfrak{N}(\varrho)}{1-\varrho} v(a) \mathbb{E}_{\varrho}\left(\frac{-\varrho}{1-\varrho}(\theta-a)^{\varrho}\right) .
$$

Lemma 2.4 ([15]) For $n<\varrho \leq n+1$, for some $n \in \mathbb{N}_{0}$ and $v(\theta)$ defined on $[0, \chi]$, we have

(i) $A B C \mathbb{D}_{a^{+}, \theta}^{\varrho} A B \mathbb{I}_{a^{+}, \theta}^{\varrho} v(\theta)=v(\theta)$;

(ii) $A B \mathbb{I}_{a^{+}, \theta}^{\varrho} A B C \mathbb{D}_{a^{+}, \theta}^{\varrho} v(\theta)=v(\theta)-\sum_{k=0}^{n} \frac{v^{(k)}(a)}{k !}(\theta-a)^{k}$;

(iii) ${ }^{A B} \mathbb{I}_{a^{+}, \theta}^{\varrho} A B R \mathbb{D}_{a^{+}, \theta}^{\varrho} v(\theta)=v(\theta)-\sum_{k=0}^{n-1} \frac{v^{(k)}(a)}{k !}(\theta-a)^{k}$.

Lemma 2.5 ([15]) For $n<\varrho \leq n+1,{ }^{A B C} \mathbb{D}_{a^{+}, \theta}^{\varrho}(\theta-a)^{k}=0, k=0,1, \ldots, n$. Moreover, $A B C \mathbb{D}_{a^{+}, \theta}^{\varrho} v(\theta)=0$ if $v(\theta)$ is a constant function.

Lemma 2.6 $([11,15])$ Let $\varrho \in(0,1]$ and $\varpi \in C[0,1]$ with $\varpi(0)=0$. Then the solution of

$$
\begin{aligned}
& { }^{A B C} \mathbb{D}_{0^{+}}^{\varrho} v(\theta)=\varpi(\theta), \quad \theta \in[0,1], \\
& v(0)=c
\end{aligned}
$$

is given by

$$
v(\theta)=c+{ }^{A B} \mathbb{I}_{0^{+}}^{\varrho} \varpi(\theta) .
$$

Theorem 2.7 ([46]) Let $\mathfrak{X}$ be a Banach space and $\mathfrak{K}$ be a nonempty closed subset of $\mathfrak{X}$. If $\mathfrak{B}: \mathfrak{K} \longrightarrow \mathfrak{K}$ is a contraction, then there exists a unique fixed point of $\mathfrak{B}$.

Theorem 2.8 ([46]) Let $\mathfrak{X}$ be a Banach space and $\mathfrak{K}$ be a convex subset of $\mathfrak{X}$ and $Q: \mathfrak{K} \rightarrow \mathfrak{K}$ be a compact and continuous map. Then $Q$ has at least one fixed point in $\mathfrak{K}$.

\section{Main results}

This section is devoted to obtaining formula of the solution to ABC-type nonlocal problem (1.1)-(1.2). Then we prove the existence and uniqueness of solution for problem (1.1)(1.2) by means of Schauder's fixed point theorem (Theorem 2.8)and Banach's fixed point theorem (Theorem 2.7). Moreover, we also discuss the continuous dependence of solutions to such equations on arbitrary data.

\subsection{Solution representation}

Lemma 3.1 Let $0<\varrho \leq 1$ and $\sum_{k=1}^{m} \beta_{k} \neq 0$. Then the solution of $A B C$-type nonlocal problem (1.1)-(1.2) can be indicated by the fractional integral equation

$$
\varkappa(\theta)=\mathcal{A}\left(\varkappa_{0}-\sum_{k=1}^{m} \beta_{k}{ }^{A B} \mathbb{I}_{0, \tau_{k}}^{\varrho} \mathfrak{F}_{\varkappa}\left(\tau_{k}\right)\right)+{ }^{A B} \mathbb{I}_{0, \theta}^{\varrho} \mathfrak{F}_{\varkappa}(\theta),
$$

where $\mathfrak{F}_{\varkappa}$ is the solution of the functional integral equation

$$
\mathfrak{F}_{\varkappa}(\theta)=f\left(\theta, \mathcal{A} \varkappa_{0}-\mathcal{A} \sum_{k=1}^{m} \beta_{k}{ }^{A B} \mathbb{I}_{0, \tau_{k}}^{\varrho} \mathfrak{F}_{\varkappa}\left(\tau_{k}\right)+{ }^{A B} \mathbb{I}_{0, \theta}^{\varrho} \mathfrak{F}_{\varkappa}(\theta), \mathfrak{F}_{\varkappa}(\theta)\right)
$$

and $\mathcal{A}:=\left(\sum_{k=1}^{m} \beta_{k}\right)^{-1}$. 
Proof Set ${ }^{A B C} \mathbb{D}_{0, \theta}^{\varrho} \varkappa(\theta)=\mathfrak{F}_{\varkappa}(\theta)$ in (1.1). Then we get

$$
\mathfrak{F}_{\varkappa}(\theta)=f\left(\theta, \varkappa(\theta), \mathfrak{F}_{\varkappa}(\theta)\right)
$$

Applying ${ }^{A B} \mathbb{I}_{0, \theta}^{\varrho}$ on both sides of (1.1) and using Lemma 2.2, we have

$$
\varkappa(\theta)=\varkappa(0)+{ }^{A B} \mathbb{I}_{0, \theta}^{\varrho} \mathfrak{F}_{\varkappa}(\theta) .
$$

Putting $\theta=\tau_{k}$ into (3.3), we get

$$
\varkappa\left(\tau_{k}\right)=\varkappa(0)+{ }^{A B} \mathbb{I}_{0, \tau_{k}}^{\varrho} \mathfrak{F}_{\varkappa}\left(\tau_{k}\right)
$$

Multiplying $\beta_{k}$ and taking the sum to both sides of (3.4), we can write

$$
\sum_{k=1}^{m} \beta_{k} \varkappa\left(\tau_{k}\right)=\sum_{k=1}^{m} \beta_{k} \varkappa(0)+\sum_{k=1}^{m} \beta_{k}^{A B} \mathbb{I}_{0, \tau_{k}}^{\varrho} \mathfrak{F}_{\varkappa}\left(\tau_{k}\right) .
$$

By nonlocal condition (1.2), we obtain

$$
\begin{aligned}
\varkappa_{0} & =\sum_{k=1}^{m} \beta_{k} \varkappa\left(\tau_{k}\right) \\
& =\sum_{k=1}^{m} \beta_{k} \varkappa(0)+\sum_{k=1}^{m} \beta_{k}{ }^{A B} \mathbb{I}_{0, \tau_{k}}^{\varrho} \mathfrak{F}_{\varkappa}\left(\tau_{k}\right),
\end{aligned}
$$

which implies

$$
\varkappa(0)=\left(\sum_{k=1}^{m} \beta_{k}\right)^{-1}\left[\varkappa_{0}-\sum_{k=1}^{m} \beta_{k}^{A B} \mathbb{I}_{0, \tau_{k}}^{\varrho} \mathfrak{F}_{\varkappa}\left(\tau_{k}\right)\right] .
$$

Since $\mathcal{A}=\left(\sum_{k=1}^{m} \beta_{k}\right)^{-1}$, we get

$$
\varkappa(\theta)=\mathcal{A}\left(\varkappa_{0}-\sum_{k=1}^{m} \beta_{k}{ }^{A B} \mathbb{I}_{0, \tau_{k}}^{\varrho} \mathfrak{F}_{\varkappa}\left(\tau_{k}\right)\right)+{ }^{A B} \mathbb{I}_{0, \theta}^{\varrho} \mathfrak{F}_{\varkappa}(\theta) .
$$

Here, $\mathfrak{F}_{\varkappa}$ is the solution of equation $\mathfrak{F}_{\varkappa}(\theta)=f\left(\theta, \varkappa(\theta), \mathfrak{F}_{\varkappa}(\theta)\right)$, i.e.,

$$
\mathfrak{F}_{\varkappa}(\theta)=f\left(\theta, \mathcal{A} \varkappa_{0}-A \sum_{k=1}^{m} \beta_{k}{ }^{A B} \mathbb{I}_{0, \tau_{k}}^{\varrho} \mathfrak{F}_{\varkappa}\left(\tau_{k}\right)+{ }^{A B} \mathbb{I}_{0, \theta}^{\varrho} \mathfrak{F}_{\varkappa}(\theta), \mathfrak{F}_{\varkappa}(\theta)\right)
$$

The proof is completed.

Now, we consider the following hypotheses:

$\left(H_{1}\right)$ There exists a constant $L_{1}>0$ such that

$$
\left|f(\theta, x, y)-f\left(\theta, x^{*}, y^{*}\right)\right| \leq L_{1}\left(\left|x-x^{*}\right|+\left|y-y^{*}\right|\right)
$$

for all $\theta \in[0, \chi]$ and $x, x^{*}, y, y^{*} \in \mathbb{R}$. 
$\left(H_{2}\right)$ There exists a constant $\kappa>0$ such that

$$
|f(\theta, x, y)| \leq \kappa(1+|x|+|y|), \quad \forall(\theta, x, y) \in[0, \chi] \times \mathbb{R} \times \mathbb{R}
$$

\subsection{Existence results}

In this subsection, we prove the existence and uniqueness of solution to ABC-type nonlocal problem (1.1)-(1.2).

The following result is based on Theorem 2.8 .

Theorem 3.2 Assume that $f:[0, \chi] \times \mathbb{R}^{2} \longrightarrow \mathbb{R}$ is continuous. If $\left(H_{2}\right)$ holds with $\kappa \neq 1$, and

$$
\eta_{1}:=\frac{\kappa}{1-\kappa}\left[\frac{\left(|\mathcal{A}| \sum_{k=1}^{m}\left|\beta_{k}\right|+1\right)(1-\varrho)}{\mathfrak{N}(\varrho)}+\frac{|\mathcal{A}| \sum_{k=1}^{m}\left|\beta_{k}\right| \tau_{k}^{\varrho}+\chi^{\varrho}}{\mathfrak{N}(\varrho) \Gamma(\varrho)}\right]<1
$$

then ABC-type nonlocal problem (1.1)-(1.2) has at least one solution $\varkappa \in C[0, \chi]$.

Proof Define the operator $\mathcal{T}: C[0, \chi] \rightarrow C[0, \chi]$ by

$$
(\mathcal{T} \varkappa)(\theta)=\mathcal{A} \varkappa_{0}-\mathcal{A} \sum_{k=1}^{m} \beta_{k}{ }^{A B} \mathbb{I}_{0, \tau_{k}}^{\varrho} \mathfrak{F}_{\varkappa}\left(\tau_{k}\right)+{ }^{A B} \mathbb{I}_{0, \theta}^{\varrho} \mathfrak{F}_{\varkappa}(\theta),
$$

where

$$
\mathfrak{F}_{\varkappa}(\theta)=f\left(\theta, \mathcal{A} \varkappa_{0}-\mathcal{A} \sum_{k=1}^{m} \beta_{k}{ }^{A B} \mathbb{I}_{0, \tau_{k}}^{\varrho} \mathfrak{F}_{\varkappa}\left(\tau_{k}\right)+{ }^{A B} \mathbb{I}_{0, \theta}^{\rho} \mathfrak{F}_{\varkappa}(\theta), \mathfrak{F}_{\varkappa}(\theta)\right) .
$$

The operator $\mathcal{T}$ is well defined. Indeed, we consider a function $\varkappa \in C[0, \chi]$. It is clear that $\mathcal{T} \varkappa \in C[0, \chi]$. Also, by equation (3.6), Lemmas 2.4 and 2.5, we have

$$
\begin{aligned}
\left({ }^{A B C} \mathbb{D}_{0, \theta}^{\varrho} \mathcal{T} \varkappa\right)(\theta)= & \mathcal{A} \varkappa_{0}\left({ }^{A B C} \mathbb{D}_{0, \theta}^{\varrho} 1\right)(\theta)-\mathcal{A} \sum_{k=1}^{m} \beta_{k}{ }^{A B} \mathbb{I}_{0, \tau_{k}}^{\varrho} \mathfrak{F}_{\varkappa}\left(\tau_{k}\right)\left({ }^{A B C} \mathbb{D}_{0, \theta}^{\varrho} 1\right)(\theta) \\
& +{ }^{A B C} \mathbb{D}_{0, \theta}^{\varrho}{ }^{A B} \mathbb{I}_{0, \theta}^{\varrho} \mathfrak{F}_{\varkappa}(\theta) \\
= & \mathfrak{F}_{\varkappa}(\theta) .
\end{aligned}
$$

Since $\varkappa \in C[0, \chi]$ and ${ }^{A B C} \mathbb{D}_{0, \theta}^{\varrho} \varkappa(\theta)=\mathfrak{F}_{\varkappa}(\theta)$ in equation (1.1), it follows that

$$
\left({ }^{A B C} \mathbb{D}_{0, \theta}^{\varrho} \mathcal{T} \varkappa\right)(\theta)=f\left(\theta, \varkappa(\theta),{ }^{A B C} \mathbb{D}_{0, \theta}^{\varrho} \varkappa(\theta)\right)
$$

As $f\left(\theta, \varkappa(\theta),{ }^{A B C} \mathbb{D}_{0, \theta}^{\varrho} \varkappa(\theta)\right)$ is continuous on $[0, \chi]$, then ${ }^{A B C} \mathbb{D}_{0, \theta}^{\varrho} \mathcal{T} \varkappa(\theta) \in C[0, \chi]$.

Let $r \geq \frac{\eta_{2}}{1-\eta_{1}}$ and $\mathcal{B}_{r}=\{\varkappa \in C[0, \chi]:\|\varkappa\| \leq r\}$, where $\mathcal{B}_{r}$ is a nonempty, closed, convex, and bounded subset of $C[0, \chi]$ and

$$
\eta_{2}=\left|\mathcal{A} \varkappa_{0}\right|+\eta_{1}
$$

Now, we show that $\mathcal{T}$ fulfills the hypotheses of Theorem 2.8. The proof is presented in numerous steps as follows. 
Step 1. $\mathcal{T} \mathcal{B}_{r} \subseteq \mathcal{B}_{r}$.

For $\theta \in[0, \chi]$, we get

$$
\begin{aligned}
|\mathcal{T} \varkappa(\theta)| \leq & \left|\mathcal{A} \varkappa_{0}\right|+|\mathcal{A}| \sum_{k=1}^{m}\left|\beta_{k}\right|{ }^{A B} \mathbb{I}_{0, \tau_{k}}^{\varrho}\left|\mathfrak{F}_{\varkappa}\left(\tau_{k}\right)\right|+{ }^{A B} \mathbb{I}_{0, \theta}^{\varrho}\left|\mathfrak{F}_{\varkappa}(\theta)\right| \\
\leq & \left|\mathcal{A} \varkappa_{0}\right|+|\mathcal{A}| \sum_{k=1}^{m}\left|\beta_{k}\right|\left[\frac{1-\varrho}{\mathfrak{N}(\varrho)}\left|\mathfrak{F}_{\varkappa}\left(\tau_{k}\right)\right|\right. \\
& \left.+\frac{\varrho}{\mathfrak{N}(\varrho)} \frac{1}{\Gamma(\varrho)} \int_{0}^{\tau_{k}}\left(\tau_{k}-\sigma\right)^{\varrho-1}\left|\mathfrak{F}_{\varkappa}(\sigma)\right| d \sigma\right] \\
& +\frac{1-\varrho}{\mathfrak{N}(\varrho)}\left|\mathfrak{F}_{\varkappa}(\theta)\right|+\frac{\varrho}{\mathfrak{N}(\varrho)} \frac{1}{\Gamma(\varrho)} \int_{0}^{\theta}(\theta-\sigma)^{\varrho-1}\left|\mathfrak{F}_{\varkappa}(\sigma)\right| d \sigma
\end{aligned}
$$

and

$$
\begin{aligned}
\left|\mathfrak{F}_{\varkappa}(\theta)\right| & =\left|f\left(\theta, \varkappa(\theta), \mathfrak{F}_{\varkappa}(\theta)\right)\right| \\
& \leq \kappa\left(1+|\varkappa(\theta)|+\left|\mathfrak{F}_{\varkappa}(\theta)\right|\right) .
\end{aligned}
$$

Thus

$$
\left|\mathfrak{F}_{\varkappa}(\theta)\right| \leq \frac{\kappa(1+|\varkappa(\theta)|)}{1-\kappa} .
$$

It follows from (3.5) and (3.8) that, for each $\varkappa \in \mathcal{B}_{r}$,

$$
\begin{aligned}
|\mathcal{T} \varkappa(\theta)| \leq & \left|\mathcal{A} \varkappa_{0}\right|+|\mathcal{A}| \frac{\kappa(1+r)}{1-\kappa} \sum_{k=1}^{m}\left|\beta_{k}\right|\left(\frac{1-\varrho}{\mathfrak{N}(\varrho)}+\frac{\tau_{k}^{\varrho}}{\mathfrak{N}(\varrho) \Gamma(\varrho)}\right) \\
& +\frac{\kappa(1+r)}{1-\kappa}\left(\frac{1-\varrho}{\mathfrak{N}(\varrho)}+\frac{\theta^{\varrho}}{\mathfrak{N}(\varrho) \Gamma(\varrho)}\right) \\
= & \left|\mathcal{A} \varkappa_{0}\right|+\frac{\kappa}{1-\kappa}\left[\left(|\mathcal{A}| \sum_{k=1}^{m}\left|\beta_{k}\right|+1\right) \frac{(1-\varrho)}{\mathfrak{N}(\varrho)}+\frac{|\mathcal{A}| \sum_{k=1}^{m}\left|\beta_{k}\right| \tau_{k}^{\varrho}+\theta^{\varrho}}{\mathfrak{N}(\varrho) \Gamma(\varrho)}\right] \\
& +\frac{\kappa}{1-\kappa}\left[\left(|\mathcal{A}| \sum_{k=1}^{m}\left|\beta_{k}\right|+1\right) \frac{(1-\varrho)}{\mathfrak{N}(\varrho)}+\frac{|\mathcal{A}| \sum_{k=1}^{m}\left|\beta_{k}\right| \tau_{k}^{\varrho}+\theta^{\varrho}}{\mathfrak{N}(\varrho) \Gamma(\varrho)}\right] r \\
\leq & \eta_{2}+\eta_{1} r \\
\leq & r .
\end{aligned}
$$

Step 2. $\mathcal{T}$ is continuous.

Let $\varkappa_{n}$ be a sequence such that $\varkappa_{n} \rightarrow \varkappa$ as $n \rightarrow \infty$. Then

$$
\begin{aligned}
& \left|\mathcal{T} \varkappa_{n}(\theta)-\mathcal{T} \varkappa(\theta)\right| \\
& \leq|\mathcal{A}| \sum_{k=1}^{m}\left|\beta_{k}\right|^{A B} \mathbb{I}_{0, \tau_{k}}^{\varrho}\left|\mathfrak{F}_{\varkappa_{n}}\left(\tau_{k}\right)-\mathfrak{F}_{\varkappa}\left(\tau_{k}\right)\right| \\
& \quad+{ }^{A B} \mathbb{I}_{0, \theta}^{\varrho}\left|\mathfrak{F}_{\varkappa_{n}}(\theta)-\mathfrak{F}_{\varkappa}(\theta)\right|
\end{aligned}
$$




$$
\begin{aligned}
\leq & |\mathcal{A}| \sum_{k=1}^{m}\left|\beta_{k}\right|\left[\frac{1-\varrho}{\mathfrak{N}(\varrho)}\left|\mathfrak{F}_{\varkappa_{n}}\left(\tau_{k}\right)-\mathfrak{F}_{\varkappa}\left(\tau_{k}\right)\right|\right. \\
& \left.+\frac{\varrho}{\mathfrak{N}(\varrho)} \frac{1}{\Gamma(\varrho)} \int_{0}^{\tau_{k}}\left(\tau_{k}-\sigma\right)^{\varrho-1}\left|\mathfrak{F}_{\varkappa_{n}}(\sigma)-\mathfrak{F}_{\varkappa}(\sigma)\right| d \sigma\right] \\
& +\frac{1-\varrho}{\mathfrak{N}(\varrho)}\left|\mathfrak{F}_{\varkappa_{n}}(\theta)-\mathfrak{F}_{\varkappa}(\theta)\right|+\frac{\varrho}{\mathfrak{N}(\varrho)} \frac{1}{\Gamma(\varrho)} \int_{0}^{\theta}(\theta-\sigma)^{\varrho-1}\left|\mathfrak{F}_{\varkappa_{n}}(\sigma)-\mathfrak{F}_{\varkappa}(\sigma)\right| d \sigma \\
\leq & \frac{\left(|\mathcal{A}| \sum_{k=1}^{m}\left|\beta_{k}\right|+1\right)(1-\varrho)}{\mathfrak{N}(\varrho)}\left\|\mathfrak{F}_{\varkappa_{n}}(\cdot)-\mathfrak{F}_{\varkappa}(\cdot)\right\| \\
& +\frac{|\mathcal{A}| \sum_{k=1}^{m}\left|\beta_{k}\right| \tau_{k}^{\varrho}+\chi^{\varrho}}{\mathfrak{N}(\varrho) \Gamma(\varrho)}\left\|\mathfrak{F}_{\varkappa_{n}}(\cdot)-\mathfrak{F}_{\varkappa}(\cdot)\right\| .
\end{aligned}
$$

Since $\mathfrak{F}_{\varkappa}(\cdot)=f\left(\cdot, \varkappa(\cdot), \mathfrak{F}_{\varkappa}(\cdot)\right) \in C[0, \chi]$, it follows that $\left\|\mathcal{T} \varkappa_{n}(\cdot)-\mathcal{T} \varkappa(\cdot)\right\| \rightarrow 0$ as $n \rightarrow \infty$, which proves the required result.

Step 3. $\mathcal{T}$ is compact.

We shall show that $\mathcal{T} \mathcal{B}_{r}$ is relatively compact. Clearly, $\mathcal{T} \mathcal{B}_{r}$ is uniformly bounded due to Step 1. It remains to show that $\mathcal{T} \mathcal{B}_{r}$ is equicontinuous. Let $\theta_{1}, \theta_{2} \in[0, \chi]$ such that $0 \leq$ $\theta_{1} \leq \theta_{2} \leq \chi$. Then

$$
\begin{aligned}
&\left|\mathcal{T} \varkappa\left(\theta_{2}\right)-\mathcal{T} \varkappa\left(\theta_{1}\right)\right| \\
&=\left|{ }^{A B} \mathbb{I}_{0, \theta_{2}}^{\varrho} \mathfrak{F}_{\varkappa}\left(\theta_{2}\right)-{ }^{A B} \mathbb{I}_{0, \theta_{1}}^{\varrho} \mathfrak{F}_{\varkappa}\left(\theta_{1}\right)\right| \\
& \leq\left|\frac{1-\varrho}{\mathfrak{N}(\varrho)}\right| \mathfrak{F}_{\varkappa}\left(\theta_{2}\right)\left|+\frac{\varrho}{\mathfrak{N}(\varrho)} \frac{1}{\Gamma(\varrho)} \int_{0}^{\theta_{2}}\left(\theta_{2}-\sigma\right)^{\varrho-1}\right| \mathfrak{F}_{\varkappa}(\sigma) \mid d \sigma \\
& \quad-\frac{1-\varrho}{\mathfrak{N}(\varrho)}\left|\mathfrak{F}_{\varkappa}\left(\theta_{1}\right)\right|-\frac{\varrho}{\mathfrak{N}(\varrho)} \frac{1}{\Gamma(\varrho)} \int_{0}^{\theta_{1}}\left(\theta_{1}-\sigma\right)^{\varrho-1}\left|\mathfrak{F}_{\varkappa}(\sigma)\right| d \sigma \mid \\
& \leq \frac{1-\varrho}{\mathfrak{N}(\varrho)}\left|\mathfrak{F}_{\varkappa}\left(\theta_{2}\right)-\mathfrak{F}_{\varkappa}\left(\theta_{1}\right)\right| \\
&+\frac{\varrho}{\mathfrak{N}(\varrho)} \frac{1}{\Gamma(\varrho)} \int_{0}^{\theta_{1}}\left|\left(\theta_{1}-\sigma\right)^{\varrho-1}-\left(\theta_{2}-\sigma\right)^{\varrho-1}\right|\left|\mathfrak{F}_{\varkappa}(\sigma)\right| d \sigma \\
&+\frac{\varrho}{\mathfrak{N}(\varrho)} \frac{1}{\Gamma(\varrho)} \int_{\theta_{1}}^{\theta_{2}}\left(\theta_{2}-\sigma\right)^{\varrho-1}\left|\mathfrak{F}_{\varkappa}(\sigma)\right| d \sigma .
\end{aligned}
$$

It follows from (3.9) that, for each $\varkappa \in \mathcal{B}_{r}$,

$$
\begin{aligned}
\left|\mathcal{T} \varkappa\left(\theta_{2}\right)-\mathcal{T} \varkappa\left(\theta_{1}\right)\right| \leq & \frac{1-\varrho}{\mathfrak{N}(\varrho)}\left|\mathfrak{F}_{\varkappa}\left(\theta_{2}\right)-\mathfrak{F}_{\varkappa}\left(\theta_{1}\right)\right| \\
& +\frac{\kappa(1+r)}{1-\kappa} \frac{\varrho}{\mathfrak{N}(\varrho)} \frac{1}{\Gamma(\varrho)} \int_{0}^{\theta_{1}}\left|\left(\theta_{1}-\sigma\right)^{\varrho-1}-\left(\theta_{2}-\sigma\right)^{\varrho-1}\right| d \sigma \\
& +\frac{\kappa(1+r)}{1-\kappa} \frac{\varrho}{\mathfrak{N}(\varrho)} \frac{1}{\Gamma(\varrho)} \int_{\theta_{1}}^{\theta_{2}}\left(\theta_{2}-\sigma\right)^{\varrho-1} d \sigma \\
= & \frac{1-\varrho}{\mathfrak{N}(\varrho)}\left|\mathfrak{F}_{\varkappa}\left(\theta_{2}\right)-\mathfrak{F}_{\varkappa}\left(\theta_{1}\right)\right| \\
& +\frac{\kappa(1+r)}{1-\kappa} \frac{1}{\mathfrak{N}(\varrho) \Gamma(\varrho)}\left[\left(\theta_{1}^{\varrho}+\left(\theta_{2}-\theta_{1}\right)^{\varrho}-\theta_{2}^{\varrho}\right]\right. \\
& +\frac{\kappa(1+r)}{1-\kappa} \frac{1}{\mathfrak{N}(\varrho) \Gamma(\varrho)}\left(\theta_{2}-\theta_{1}\right)^{\varrho}
\end{aligned}
$$




$$
\leq \frac{1-\varrho}{\mathfrak{N}(\varrho)}\left|\mathfrak{F}_{\varkappa}\left(\theta_{2}\right)-\mathfrak{F}_{\varkappa}\left(\theta_{1}\right)\right|+\frac{\kappa(1+r)}{1-\kappa} \frac{2\left(\theta_{2}-\theta_{1}\right)^{\varrho}}{\mathfrak{N}(\varrho) \Gamma(\varrho)}
$$

Since $\mathfrak{F}_{\varkappa}(\cdot)=f\left(\cdot, \varkappa(\cdot), \mathfrak{F}_{\varkappa}(\cdot)\right) \in C\left[\theta_{1}, \theta_{2}\right]$, it follows that $\left|\mathcal{T} \varkappa\left(\theta_{2}\right)-\mathcal{T} \varkappa\left(\theta_{1}\right)\right| \rightarrow 0$ as $\theta_{2} \rightarrow \theta_{1}$. As a result of Steps 1 to 3 together with the Arzela-Ascoli theorem, we arrive at $\mathcal{T}$ being continuous and compact. According to Theorem 2.8, ABC-type nonlocal problem (1.1)(1.2) has at least one solution in $\mathcal{B}_{r}$.

The following result is based on Theorem 2.7.

Theorem 3.3 Assume that $f:[0, \chi] \times \mathbb{R}^{2} \longrightarrow \mathbb{R}$ is continuous. If $\left(H_{1}\right)$ holds with $L_{1} \neq 1$, then ABC-type nonlocal problem (1.1)-(1.2) has a unique solution $\varkappa \in C[0, \chi]$ provided that

$$
\Upsilon:=\frac{L_{1}}{1-L_{1}}\left[\frac{\left(|\mathcal{A}| \sum_{k=1}^{m}\left|\beta_{k}\right|+1\right)(1-\varrho)}{\mathfrak{N}(\varrho)}+\frac{|\mathcal{A}| \sum_{k=1}^{m}\left|\beta_{k}\right| \tau_{k}{ }^{\varrho}+\chi^{\varrho}}{\mathfrak{N}(\varrho) \Gamma(\varrho)}\right]<1 .
$$

Proof We shall use Theorem 2.7 to prove that $\mathcal{T}$ defined by (3.6) has a fixed point.

Let $\varkappa, \varkappa^{*} \in C[0, \chi]$ and $\theta \in[0, \chi]$. Then

$$
\begin{aligned}
\left|(\mathcal{T} \varkappa)(\theta)-\left(\mathcal{T} \varkappa^{*}\right)(\theta)\right| \leq & |\mathcal{A}| \sum_{k=1}^{m}\left|\beta_{k}\right|^{A B} \mathbb{I}_{0, \tau_{k}}^{\varrho}\left|\mathfrak{F}_{\varkappa}\left(\tau_{k}\right)-\mathfrak{F}_{\varkappa^{*}}\left(\tau_{k}\right)\right| \\
& +{ }^{A B} \mathbb{I}_{0, \theta}^{\varrho}\left|\mathfrak{F}_{\varkappa}(\theta)-\mathfrak{F}_{\varkappa^{*}}(\theta)\right| .
\end{aligned}
$$

On the other hand, we have, for each $\theta \in[0, \chi]$,

$$
\begin{aligned}
\left|\mathfrak{F}_{\varkappa}(\theta)-\mathfrak{F}_{\varkappa^{*}}(\theta)\right| & =\left|f\left(\theta, \varkappa(\theta), \mathfrak{F}_{\varkappa}(\theta)\right)-f\left(\theta, \varkappa^{*}(\theta), \mathfrak{F}_{\varkappa^{*}}(\theta)\right)\right| \\
& \leq L_{1}\left(\left|\varkappa(\theta)-\varkappa^{*}(\theta)\right|+\left|\mathfrak{F}_{\varkappa}(\theta)-\mathfrak{F}_{\varkappa^{*}}(\theta)\right|\right) .
\end{aligned}
$$

Thus

$$
\left|\mathfrak{F}_{\varkappa}(\theta)-\mathfrak{F}_{\varkappa^{*}}(\theta)\right| \leq \frac{L_{1}}{1-L_{1}}\left|\varkappa(\theta)-\varkappa^{*}(\theta)\right|
$$

By replacing (3.12) in (3.11), we get

$$
\begin{aligned}
\left|(\mathcal{T} \varkappa)(\theta)-\left(\mathcal{T} \varkappa^{*}\right)(\theta)\right| \leq & \frac{|\mathcal{A}| L_{1}}{1-L_{1}} \sum_{k=1}^{m}\left|\beta_{k}\right|^{A B} \mathbb{I}_{0, \tau_{k}}^{\varrho}\left|\varkappa\left(\tau_{k}\right)-\varkappa^{*}\left(\tau_{k}\right)\right| \\
& +\frac{L_{1}}{1-L_{1}}{ }^{A B} \mathbb{I}_{0, \theta}^{\varrho}\left|\varkappa(\theta)-\varkappa^{*}(\theta)\right| \\
= & \frac{|\mathcal{A}| L_{1}}{1-L_{1}} \sum_{k=1}^{m}\left|\beta_{k}\right|\left[\frac{1-\varrho}{\mathfrak{N}(\varrho)}\left|\varkappa\left(\tau_{k}\right)-\varkappa^{*}\left(\tau_{k}\right)\right|\right. \\
& \left.+\frac{\varrho}{\mathfrak{N}(\varrho)} \frac{1}{\Gamma(\varrho)} \int_{0}^{\tau_{k}}\left(\tau_{k}-\sigma\right)^{\varrho-1}\left|\varkappa(\sigma)-\varkappa^{*}(\sigma)\right| d \sigma\right] \\
& +\frac{L_{1}}{1-L_{1}}\left[\frac{1-\varrho}{\mathfrak{N}(\varrho)}\left|\varkappa(\theta)-\varkappa^{*}(\theta)\right|\right.
\end{aligned}
$$




$$
\begin{aligned}
& \left.+\frac{\varrho}{\mathfrak{N}(\varrho)} \frac{1}{\Gamma(\varrho)} \int_{0}^{\theta}(\theta-\sigma)^{\varrho-1}\left|\varkappa(\sigma)-\varkappa^{*}(\sigma)\right| d \sigma\right] \\
\leq & \frac{L_{1}}{1-L_{1}}\left[\frac{\left(|\mathcal{A}| \sum_{k=1}^{m}\left|\beta_{k}\right|+1\right)(1-\varrho)}{\mathfrak{N}(\varrho)}\right. \\
& \left.+\frac{|\mathcal{A}| \sum_{k=1}^{m}\left|\beta_{k}\right| \tau_{k} \varrho+\chi^{\varrho}}{\mathfrak{N}(\varrho) \Gamma(\varrho)}\right]\left\|\varkappa-\varkappa^{*}\right\| .
\end{aligned}
$$

Consequently, by (3.10), $\mathcal{T}$ is a contraction. As a consequence of Theorem 2.7, we conclude that $\mathcal{T}$ has a fixed point which is a solution of problem (1.1)-(1.2).

\subsection{Continuous dependence}

This portion is devoted to discussing the continuous dependence of the solution for $\mathrm{ABC}$ type nonlocal problem (1.1)-(1.2).

Definition 3.4 The solution $\varkappa \in C[0, \chi]$ of ABC-type nonlocal problem (1.1)-(1.2) is called continuously dependent on $\varkappa_{0}$ if, for every $\epsilon>0$, there exists $\delta(\epsilon)>0$ such that $\left|\varkappa_{0}-\tilde{\varkappa}_{0}\right|<\delta$ implies $\|\varkappa-\tilde{\varkappa}\|<\epsilon$, where $\tilde{\varkappa}$ is the solution of equation (1.1) with the nonlocal condition

$$
\sum_{k=1}^{m} \beta_{k} \varkappa\left(\tau_{k}\right)=\tilde{\varkappa}_{0}, \quad \tau_{k} \in(0, \chi)
$$

Theorem 3.5 Assume that the hypotheses of Theorem 3.3 are fulfilled. Then the solution of ABC-type nonlocal problem (1.1)-(1.2) depends continuously on $\varkappa_{0}$.

Proof In view of Lemma 3.1, the solution of ABC-type nonlocal problem (1.1)-(1.2) is

$$
\varkappa(\theta)=\mathcal{A}\left(\varkappa_{0}-\sum_{k=1}^{m} \beta_{k}{ }^{A B} \mathbb{I}_{0, \tau_{k}}^{\varrho} \mathfrak{F}_{\varkappa}\left(\tau_{k}\right)\right)+{ }^{A B} \mathbb{I}_{0, \theta}^{\varrho} \mathfrak{F}_{\varkappa}(\theta),
$$

and the solution of ABC-type nonlocal problem (1.1)-(3.13) is

$$
\tilde{\varkappa}(\theta)=\mathcal{A}\left(\tilde{\varkappa}_{0}-\sum_{k=1}^{m} \beta_{k}{ }^{A B} \mathbb{I}_{0, \tau_{k}}^{\varrho} \mathfrak{F} \widetilde{\varkappa}_{(}\left(\tau_{k}\right)\right)+{ }^{A B} \mathbb{I}_{0, \theta}^{\varrho} \mathfrak{F}_{\tilde{\varkappa}}(\theta),
$$

where $\mathfrak{F}_{\varkappa}$ and $\mathfrak{F}_{\varkappa}$ are the solutions of

$$
\begin{aligned}
\mathfrak{F}_{\varkappa}(\theta) & =f\left(\theta, \mathcal{A} \varkappa_{0}-\mathcal{A} \sum_{k=1}^{m} \beta_{k}{ }^{A B} \mathbb{I}_{0, \tau_{k}}^{\varrho} \mathfrak{F}_{\varkappa}\left(\tau_{k}\right)+{ }^{A B} \mathbb{I}_{0, \theta}^{\varrho} \mathfrak{F}_{\varkappa}(\theta), \mathfrak{F}_{\varkappa}(\theta)\right) \\
& =f\left(\theta, \varkappa(\theta), \mathfrak{F}_{\varkappa}(\theta)\right)
\end{aligned}
$$

and

$$
\begin{aligned}
\mathfrak{F}_{\varkappa}(\theta) & =f\left(\theta, \mathcal{A} \tilde{\varkappa}_{0}-\mathcal{A} \sum_{k=1}^{m} \beta_{k}{ }^{A B} \mathbb{I}_{0, \tau_{k}}^{\varrho} \mathfrak{F}_{\tilde{\varkappa}}\left(\tau_{k}\right)+{ }^{A B} \mathbb{I}_{0, \theta}^{\varrho} \mathfrak{F} \widetilde{\varkappa}_{(\theta), \mathfrak{F} \widetilde{\varkappa}(\theta))}\right. \\
& =f(\theta, \tilde{\varkappa}(\theta), \mathfrak{F} \widetilde{\varkappa}(\theta)) .
\end{aligned}
$$


Hence,

$$
\begin{aligned}
|\varkappa(\theta)-\tilde{\varkappa}(\theta)| \leq & |\mathcal{A}|\left(\left|\varkappa_{0}-\tilde{\varkappa}_{0}\right|+\sum_{k=1}^{m}\left|\beta_{k}\right|^{A B} \mathbb{I}_{0, \tau_{k}}^{\varrho}\left|\mathfrak{F}_{\varkappa}\left(\tau_{k}\right)-\mathfrak{F}_{\tilde{\varkappa}}\left(\tau_{k}\right)\right|\right) \\
& +{ }^{A B} \mathbb{I}_{0, \theta}^{\varrho}\left|\mathfrak{F}_{\varkappa}(\theta)-\mathfrak{F} \tilde{\varkappa}(\theta)\right| .
\end{aligned}
$$

However, we have from $\left(H_{1}\right)$ that

$$
\begin{aligned}
\left|\mathfrak{F}_{\varkappa}(\theta)-\mathfrak{F}_{\tilde{\varkappa}}(\theta)\right| & \leq\left|f\left(\theta, \varkappa(\theta), \mathfrak{F}_{\varkappa}(\theta)\right)-f\left(\theta, \tilde{\varkappa}(\theta), \mathfrak{F}_{\tilde{\varkappa}}(\theta)\right)\right| \\
& \leq L_{1}|\varkappa(\theta)-\tilde{\varkappa}(\theta)|+L_{1}\left|\mathfrak{F}_{\varkappa}(\theta)-\mathfrak{F}_{\varkappa}(\theta)\right| .
\end{aligned}
$$

Thus

$$
\left|\mathfrak{F}_{\varkappa}(\theta)-\mathfrak{F}_{\tilde{\varkappa}}(\theta)\right| \leq \frac{L_{1}}{1-L_{1}}|\varkappa(\theta)-\tilde{\varkappa}(\theta)| .
$$

By replacing (3.17) in (3.16), we get

$$
\begin{aligned}
& |\varkappa(\theta)-\tilde{\varkappa}(\theta)| \leq|\mathcal{A}|\left(\left|\varkappa_{0}-\tilde{\varkappa}_{0}\right|+\frac{L_{1}}{1-L_{1}} \sum_{k=1}^{m}\left|\beta_{k}\right|^{A B} \mathbb{I}_{0, \tau_{k}}^{\varrho}|\varkappa(\theta)-\tilde{\varkappa}(\theta)|\right) \\
& +\frac{L_{1}}{1-L_{1}}{ }^{A B} \mathbb{I}_{0, \theta}^{\varrho}|\varkappa(\theta)-\tilde{\varkappa}(\theta)| \\
& \leq|\mathcal{A}|\left|\varkappa_{0}-\tilde{\varkappa}_{0}\right|+\frac{L_{1}}{1-L_{1}}|\mathcal{A}| \sum_{k=1}^{m}\left|\beta_{k}\right| \frac{1-\varrho}{\mathfrak{N}(\varrho)}\|\varkappa-\tilde{\varkappa}\| \\
& +\frac{L_{1}}{1-L_{1}}|\mathcal{A}| \sum_{k=1}^{m}\left|\beta_{k}\right| \frac{\tau_{k}^{\varrho}}{\mathfrak{N}(\varrho) \Gamma(\varrho)}\|\varkappa-\tilde{\varkappa}\| \\
& +\frac{L_{1}}{1-L_{1}} \frac{1-\varrho}{\mathfrak{N}(\varrho)}\|\varkappa-\tilde{\varkappa}\|+\frac{L_{1}}{1-L_{1}} \frac{\theta^{\varrho}}{\mathfrak{N}(\varrho) \Gamma(\varrho)}\|\varkappa-\tilde{\varkappa}\| \\
& \leq|\mathcal{A}|\left|\varkappa_{0}-\tilde{\varkappa}_{0}\right|+\frac{L_{1}}{1-L_{1}} \frac{(1-\varrho)\left(|\mathcal{A}| \sum_{k=1}^{m}\left|\beta_{k}\right|+1\right)}{\mathfrak{N}(\varrho)}\|\varkappa-\tilde{\varkappa}\| \\
& +\frac{L_{1}}{1-L_{1}} \frac{|\mathcal{A}| \sum_{k=1}^{m}\left|\beta_{k}\right| \tau_{k}^{\varrho}+\theta^{\varrho}}{\mathfrak{N}(\varrho) \Gamma(\varrho)}\|\varkappa-\tilde{\varkappa}\| \\
& \leq|\mathcal{A}|\left|\varkappa_{0}-\tilde{\varkappa}_{0}\right|+\frac{L_{1}}{1-L_{1}}\left[\frac{\left(|\mathcal{A}| \sum_{k=1}^{m}\left|\beta_{k}\right|+1\right)(1-\varrho)}{\mathfrak{N}(\varrho)}\right. \\
& \left.+\frac{|\mathcal{A}| \sum_{k=1}^{m}\left|\beta_{k}\right| \tau_{k}^{\varrho}+\chi^{\varrho}}{\mathfrak{N}(\varrho) \Gamma(\varrho)}\right]\left\|\varkappa-\varkappa^{*}\right\| \text {. }
\end{aligned}
$$

Since $\Upsilon<1$, we get

$$
\|\varkappa-\tilde{\varkappa}\| \leq \frac{|\mathcal{A}|}{1-\Upsilon}\left|\varkappa_{0}-\tilde{\varkappa}_{0}\right|<\frac{|\mathcal{A}|}{1-\Upsilon} \delta=\epsilon .
$$

Definition 3.6 The solution $\varkappa \in C[0, \chi]$ of ABC-type nonlocal problem (1.1)-(1.2) is called continuously dependent on the coefficients $\sum_{k=1}^{m} \beta_{k}$ if, for every $\epsilon>0$, there exists $\delta(\epsilon)>0$ such that $\sum_{k=1}^{m}\left|\beta_{k}-\widetilde{\beta}_{k}\right|<\delta$ implies $\|\varkappa-\tilde{\varkappa}\|<\epsilon$, where $\tilde{\varkappa}$ is the solution of 
equation (1.1) with the nonlocal condition

$$
\sum_{k=1}^{m} \widetilde{\beta_{k}} \varkappa\left(\tau_{k}\right)=\varkappa_{0}, \quad \tau_{k} \in(0, \chi)
$$

Theorem 3.7 Assume that the hypotheses of Theorem 3.3 hold. Then the solution of ABCtype nonlocal problem (1.1)-(1.2) depends continuously on the coefficients $\sum_{k=1}^{m} \beta_{k}$, provided that

$$
\begin{aligned}
& \left|\frac{1}{\sum_{k=1}^{m} \beta_{k} \sum_{k=1}^{m} \widetilde{\beta_{k}}}\right| \leq 1, \\
& \Theta_{1}:=L_{1}\left(1+|\widetilde{\mathcal{A}}| \sum_{k=1}^{m}\left|\widetilde{\beta_{k}}\right| \frac{\tau_{k}^{\varrho}}{\mathfrak{N}(\varrho) \Gamma(\varrho)}\right)<1,
\end{aligned}
$$

and

$$
\Theta_{2}:=\left(|\mathcal{A}| \sum_{k=1}^{m}\left|\beta_{k}\right|+1\right)\left(\frac{1-\varrho}{\mathfrak{N}(\varrho)}+\frac{|\mathcal{A}| \sum_{k=1}^{m}\left|\beta_{k}\right| \tau_{k}^{\varrho}+\chi^{\varrho}}{\mathfrak{N}(\varrho) \Gamma(\varrho)}\right) \frac{L_{1}}{1-L_{1}}<1
$$

where $\widetilde{\mathcal{A}}=\frac{1}{\sum_{k=1}^{m} \beta_{k}}$ and $\sum_{k=1}^{m} \widetilde{\beta_{k}} \neq 0$.

Proof In view of Lemma 3.1, the solution of ABC-type nonlocal problem (1.1)-(1.2) is

$$
\varkappa(\theta)=\mathcal{A}\left(\varkappa_{0}-\sum_{k=1}^{m} \beta_{k}{ }^{A B} \mathbb{I}_{0, \tau_{k}}^{\varrho} \mathfrak{F}_{\varkappa}\left(\tau_{k}\right)\right)+{ }^{A B} \mathbb{I}_{0, \theta}^{\varrho} \mathfrak{F}_{\varkappa}(\theta),
$$

and the solution of ABC-type nonlocal problem (1.1)-(3.18) is

$$
\tilde{\varkappa}(\theta)=\widetilde{\mathcal{A}}\left(\varkappa_{0}-\sum_{k=1}^{m}{\widetilde{\beta_{k}}}^{A B} \mathbb{I}_{0, \tau_{k}}^{\varrho} \mathfrak{F}_{\tilde{\varkappa}}\left(\tau_{k}\right)\right)+{ }^{A B} \mathbb{I}_{0, \theta}^{\varrho} \mathfrak{F}_{\tilde{\varkappa}}(\theta),
$$

where $\mathfrak{F}_{\varkappa}$ and $\mathfrak{F}_{\varkappa}$ are the solutions of

$$
\begin{aligned}
& \mathfrak{F}_{\varkappa}(\theta)=f\left(\theta, \mathcal{A} \varkappa_{0}-A \sum_{k=1}^{m} \beta_{k}{ }^{A B} \mathbb{I}_{0, \tau_{k}}^{\varrho} \mathfrak{F}_{\varkappa}\left(\tau_{k}\right)+{ }^{A B} \mathbb{I}_{0, \theta}^{\varrho} \mathfrak{F}_{\varkappa}(\theta), \mathfrak{F}_{\varkappa}(\theta)\right), \\
& \mathfrak{F}_{\tilde{\varkappa}}(\theta)=f\left(\theta, \widetilde{\mathcal{A}} \varkappa_{0}-\widetilde{\mathcal{A}} \sum_{k=1}^{m}{\widetilde{\beta_{k}}}^{A B} \mathbb{I}_{0, \tau_{k}}^{\varrho} \mathfrak{F}_{\tilde{\varkappa}}\left(\tau_{k}\right)+{ }^{A B} \mathbb{I}_{0, \theta}^{\varrho} \mathfrak{F}_{\tilde{\varkappa}}(\theta), \mathfrak{F}_{\tilde{\varkappa}}(\theta)\right) .
\end{aligned}
$$

Hence,

$$
\begin{aligned}
\varkappa(\theta)-\tilde{\varkappa}(\theta)= & \varkappa_{0}(\mathcal{A}-\widetilde{\mathcal{A}})-\mathcal{A} \sum_{k=1}^{m} \beta_{k}{ }^{A B} \mathbb{I}_{0, \tau_{k}}^{\varrho} \mathfrak{F}_{\varkappa}\left(\tau_{k}\right) \\
& +\widetilde{\mathcal{A}} \sum_{k=1}^{m} \widetilde{\beta}_{k}{ }^{A B} \mathbb{I}_{0, \tau_{k}}^{\varrho} \mathfrak{F}_{\tilde{\varkappa}}\left(\tau_{k}\right)+{ }^{A B} \mathbb{I}_{0, \theta}^{\varrho}\left(\mathfrak{F}_{\varkappa}(\theta)-\mathfrak{F}_{\varkappa}(\theta)\right) \\
= & \varkappa_{0}(\mathcal{A}-\widetilde{\mathcal{A}})-\mathcal{J}+\widetilde{\mathcal{J}}+{ }^{A B} \mathbb{I}_{0, \theta}^{\varrho}\left(\mathfrak{F}_{\varkappa}(\theta)-\mathfrak{F} \widetilde{\varkappa}(\theta)\right) .
\end{aligned}
$$


Since

$$
\begin{aligned}
& \mathcal{J}-\tilde{\mathcal{J}}=\mathcal{A} \sum_{k=1}^{m} \beta_{k}{ }^{A B} \mathbb{I}_{0, \tau_{k}}^{\varrho} \mathfrak{F}_{\varkappa}\left(\tau_{k}\right)-\widetilde{\mathcal{A}} \sum_{k=1}^{m} \widetilde{\beta}_{k}{ }^{A B} \mathbb{I}_{0, \tau_{k}}^{\varrho} \mathfrak{F}_{\varkappa}\left(\tau_{k}\right) \\
& =\mathcal{A} \sum_{k=1}^{m} \beta_{k}{ }^{A B} \mathbb{I}_{0, \tau_{k}}^{\varrho} \mathfrak{F}_{\varkappa}\left(\tau_{k}\right)-\mathcal{A} \sum_{k=1}^{m} \beta_{k}{ }^{A B} \mathbb{I}_{0, \tau_{k}}^{\varrho} \mathfrak{F} \widetilde{\varkappa}_{\varkappa}\left(\tau_{k}\right) \\
& +\mathcal{A} \sum_{k=1}^{m} \beta_{k}^{A B} \mathbb{I}_{0, \tau_{k}}^{\varrho} \mathfrak{F} \widetilde{\varkappa}\left(\tau_{k}\right)-\mathcal{A} \sum_{k=1}^{m}{\widetilde{\beta_{k}}}^{A B} \mathbb{I}_{0, \tau_{k}}^{\varrho} \mathfrak{F} \widetilde{\varkappa}\left(\tau_{k}\right) \\
& +\mathcal{A} \sum_{k=1}^{m}{\widetilde{\beta_{k}}}^{A B} \mathbb{I}_{0, \tau_{k}}^{\varrho} \mathfrak{F} \tilde{\varkappa}\left(\tau_{k}\right)-\widetilde{\mathcal{A}} \sum_{k=1}^{m}{\widetilde{\beta_{k}}}^{A B} \mathbb{I}_{0, \tau_{k}}^{\varrho} \mathfrak{F} \tilde{\varkappa}\left(\tau_{k}\right) \\
& =\mathcal{A} \sum_{k=1}^{m} \beta_{k}^{A B} \mathbb{I}_{0, \tau_{k}}^{\varrho}\left(\mathfrak{F}_{\varkappa}\left(\tau_{k}\right)-\mathfrak{F}_{\varkappa}\left(\tau_{k}\right)\right) \\
& \left.+\mathcal{A} \sum_{k=1}^{m}\left(\beta_{k}-\widetilde{\beta}_{k}\right)^{A B} \mathbb{I}_{0, \tau_{k}}^{\varrho} \mathfrak{F} \widetilde{\varkappa}\left(\tau_{k}\right)+(\mathcal{A}-\widetilde{\mathcal{A}}) \sum_{k=1}^{m}{\widetilde{\beta_{k}}}^{A B} \mathbb{I}_{0, \tau_{k}}^{\varrho} \mathfrak{F} \widetilde{\varkappa}_{(}\right)
\end{aligned}
$$

Substituting from (3.21) in (3.20), we get

$$
\begin{aligned}
\varkappa(\theta)-\tilde{\varkappa}(\theta)= & \varkappa_{0}(\mathcal{A}-\widetilde{\mathcal{A}})-\mathcal{A} \sum_{k=1}^{m} \beta_{k}{ }^{A B} \mathbb{I}_{0, \tau_{k}}^{\varrho}\left(\mathfrak{F}_{\varkappa}\left(\tau_{k}\right)-\mathfrak{F}_{\tilde{\varkappa}^{\prime}}\left(\tau_{k}\right)\right) \\
& -\mathcal{A} \sum_{k=1}^{m}\left(\beta_{k}-\widetilde{\beta}_{k}\right)^{A B} \mathbb{I}_{0, \tau_{k}}^{\varrho} \mathfrak{F} \tilde{\varkappa}_{(}\left(\tau_{k}\right)-(\mathcal{A}-\widetilde{\mathcal{A}}) \sum_{k=1}^{m} \widetilde{\beta}_{k}{ }^{A B} \mathbb{I}_{0, \tau_{k}}^{\varrho} \mathfrak{F}_{\varkappa}\left(\tau_{k}\right) \\
& +{ }^{A B} \mathbb{I}_{0, \theta}^{\varrho}\left(\mathfrak{F}_{\varkappa}(\theta)-\mathfrak{F}_{\varkappa}(\theta)\right) .
\end{aligned}
$$

Then

$$
\begin{aligned}
& \|\varkappa-\tilde{\varkappa}\| \\
& \leq\left|\varkappa_{0}\right||\mathcal{A}-\tilde{\mathcal{A}}|+|\mathcal{A}|\left|\sum_{k=1}^{m} \beta_{k}\right|\left(\frac{1-\varrho}{\mathfrak{N}(\varrho)}\left\|\mathfrak{F}_{\varkappa}-\mathfrak{F}_{\tilde{\varkappa}}\right\|\right. \\
& \left.+\frac{\varrho}{\mathfrak{N}(\varrho)} \frac{1}{\Gamma(\varrho)} \int_{0}^{\tau_{k}}\left(\tau_{k}-\sigma\right)^{\varrho-1}\left\|\mathfrak{F}_{\varkappa}-\mathfrak{F} \tilde{\varkappa}\right\| d \sigma\right) \\
& +|\mathcal{A}|\left|\sum_{k=1}^{m}\left(\beta_{k}-\widetilde{\beta}_{k}\right)\right|\left(\frac{1-\varrho}{\mathfrak{N}(\varrho)}\left\|\mathfrak{F}_{\tilde{\varkappa}}\right\|+\frac{\varrho}{\mathfrak{N}(\varrho)} \frac{1}{\Gamma(\varrho)} \int_{0}^{\tau_{k}}\left(\tau_{k}-\sigma\right)^{\varrho-1}\|\mathfrak{F} \tilde{\varkappa}\| d \sigma\right) \\
& +|\mathcal{A}-\widetilde{\mathcal{A}}|\left|\sum_{k=1}^{m} \widetilde{\beta_{k}}\right|\left(\frac{1-\varrho}{\mathfrak{N}(\varrho)}\left\|\mathfrak{F}_{\tilde{\varkappa}}\right\|+\frac{\varrho}{\mathfrak{N}(\varrho)} \frac{1}{\Gamma(\varrho)} \int_{0}^{\tau_{k}}\left(\tau_{k}-\sigma\right)^{\varrho-1}\|\mathfrak{F} \tilde{\varkappa}\| d \sigma\right) \\
& +\frac{1-\varrho}{\mathfrak{N}(\varrho)}\left\|\mathfrak{F}_{\varkappa}-\mathfrak{F} \tilde{\varkappa}\right\|+\frac{\varrho}{\mathfrak{N}(\varrho)} \frac{1}{\Gamma(\varrho)} \int_{0}^{\theta}(\theta-\sigma)^{\varrho-1}\left\|\mathfrak{F}_{\varkappa}-\mathfrak{F}_{\varkappa}\right\| d \sigma \\
& \leq\left|\varkappa_{0}\right||\mathcal{A}-\widetilde{\mathcal{A}}|+\left(|\mathcal{A}| \sum_{k=1}^{m}\left|\beta_{k}\right|+1\right)\left(\frac{1-\varrho}{\mathfrak{N}(\varrho)}+\frac{|\mathcal{A}| \sum_{k=1}^{m}\left|\beta_{k}\right| \tau_{k}^{\varrho}+\theta^{\varrho}}{\mathfrak{N}(\varrho) \Gamma(\varrho)}\right)\left\|\mathfrak{F}_{\varkappa}-\mathfrak{F}_{\varkappa}\right\| \\
& +\left(|\mathcal{A}|\left|\sum_{k=1}^{m}\left(\beta_{k}-\widetilde{\beta}_{k}\right)\right|+|\mathcal{A}-\widetilde{\mathcal{A}}|\left|\sum_{k=1}^{m} \widetilde{\beta}_{k}\right|\right)\left(\frac{1-\varrho}{\mathfrak{N}(\varrho)}+\frac{\tau_{k}^{\varrho}}{\mathfrak{N}(\varrho) \Gamma(\varrho)}\right)\|\mathfrak{F} \tilde{\varkappa}\| .
\end{aligned}
$$


Now, we have from (3.19) that

$$
|\mathcal{A}-\widetilde{\mathcal{A}}|=\left|\frac{1}{\sum_{k=1}^{m} \beta_{k}}-\frac{1}{\sum_{k=1}^{m} \widetilde{\beta}_{k}}\right|=\left|\frac{\sum_{k=1}^{m} \widetilde{\beta}_{k}-\beta_{k}}{\sum_{k=1}^{m} \beta_{k} \sum_{k=1}^{m} \widetilde{\beta}_{k}}\right| \leq \sum_{k=1}^{m}\left|\beta_{k}-\widetilde{\beta}_{k}\right| \leq \delta .
$$

By $\left(H_{1}\right)$, we obtain

$$
\left\|\mathfrak{F}_{\varkappa}-\mathfrak{F}_{\varkappa}\right\| \leq \frac{L_{1}}{1-L_{1}}\|\varkappa-\tilde{\varkappa}\|
$$

and

$$
\begin{aligned}
& \left\|\mathfrak{F}_{\tilde{\varkappa}}\right\| \leq \frac{L_{1}\|\tilde{\varkappa}\|+\left\|\mathfrak{F}_{0}\right\|}{1-L_{1}} \\
& \leq \frac{L_{1}\left|\widetilde{\mathcal{A}} \varkappa_{0}\right|+\left\|\mathfrak{F}_{0}\right\|}{1-L_{1}}+\frac{L_{1}\left|\widetilde{\mathcal{A}} \sum_{k=1}^{m} \widetilde{\beta}_{k} \frac{\tau_{k}^{\varrho}}{\mathfrak{N}(\varrho) \Gamma(\varrho)}\right|}{1-L_{1}}\left\|\mathfrak{F}_{\tilde{\varkappa}}\right\|,
\end{aligned}
$$

which gives

$$
\left\|\mathfrak{F}_{\varkappa}\right\| \leq \frac{L_{1}|\widetilde{\mathcal{A}}|\left|\varkappa_{0}\right|+\left\|\mathfrak{F}_{0}\right\|}{1-\Theta_{1}}
$$

where $\left\|\mathfrak{F}_{0}\right\|=\max _{\theta \in[0, \chi]}|f(\theta, 0,0)|$. By replacing (3.23), (3.24), and (3.25) in (3.22), we obtain

$$
\begin{aligned}
\|\varkappa-\tilde{\varkappa}\| \leq & \left|\varkappa_{0}\right| \delta+\Theta_{2}\|\varkappa-\tilde{\varkappa}\|+\left(|\mathcal{A}|\left|\sum_{k=1}^{m}\left(\beta_{k}-\widetilde{\beta}_{k}\right)\right|+|\mathcal{A}-\widetilde{\mathcal{A}}|\left|\sum_{k=1}^{m} \widetilde{\beta}_{k}\right|\right) \\
& \times\left(\frac{1-\varrho}{\mathfrak{N}(\varrho)}+\frac{\tau_{k}^{\varrho}}{\mathfrak{N}(\varrho) \Gamma(\varrho)}\right) \frac{\left(L_{1}|\tilde{\mathcal{A}}|\left|\varkappa_{0}\right|+\left\|\mathfrak{F}_{0}\right\|\right)}{\left(1-\Theta_{1}\right)} \\
= & \left|\varkappa_{0}\right| \delta+\Theta_{2}\|\varkappa-\tilde{\varkappa}\|+\Theta_{3},
\end{aligned}
$$

which implies

$$
\|\varkappa-\tilde{\varkappa}\| \leq \frac{\left|\varkappa_{0}\right| \delta+\Theta_{3}}{1-\Theta_{2}}=\epsilon,
$$

where

$$
\begin{aligned}
\Theta_{3}:= & \left(|\mathcal{A}|\left|\sum_{k=1}^{m}\left(\beta_{k}-\widetilde{\beta}_{k}\right)\right|+|\mathcal{A}-\widetilde{\mathcal{A}}|\left|\sum_{k=1}^{m} \widetilde{\beta}_{k}\right|\right) \\
& \times\left(\frac{1-\varrho}{\mathfrak{N}(\varrho)}+\frac{\tau_{k}^{\varrho}}{\mathfrak{N}(\varrho) \Gamma(\varrho)}\right) \frac{\left(L_{1}|\widetilde{\mathcal{A}}|\left|\varkappa_{0}\right|+\left\|\mathfrak{F}_{0}\right\|\right)}{\left(1-\Theta_{1}\right)} .
\end{aligned}
$$

\section{Examples}

Example 4.1 Let us consider the following ABC-type nonlocal problem:

$$
A B C \mathbb{D}_{0^{+}}^{\frac{1}{3}} \varkappa(\theta)=\frac{\theta^{2}}{8}\left(1+\frac{|\varkappa(\theta)|+\left.\right|^{A B C} \mathbb{D}_{0^{+}}^{\frac{1}{3}} \varkappa(\theta) \mid}{1+|\varkappa(\theta)|+\left|{ }^{A B C} \mathbb{D}_{0^{+}}^{\frac{1}{3}} \varkappa(\theta)\right|}\right), \quad \theta \in\left[0, \frac{1}{2}\right]
$$


with nonlocal conditions

$$
\frac{1}{8} \varkappa\left(\frac{1}{6}\right)+\frac{3}{8} \varkappa\left(\frac{1}{4}\right)+\frac{1}{2} \varkappa\left(\frac{1}{3}\right)=1
$$

where $0<\left(\tau_{1}=\frac{1}{6}, \tau_{2}=\frac{1}{4}, \tau_{3}=\frac{1}{3}\right)<\frac{1}{2},\left(\beta_{1}=\frac{1}{8}, \beta_{2}=\frac{3}{8}, \beta_{3}=\frac{1}{2}\right)>0(k=1,2,3)(m=3), \varrho=\frac{1}{3}$.

Notice that (4.1)-(4.2) is a particular case of (1.1)-(1.2).

Set $f(\theta, \varkappa, v)=\frac{\theta^{2}}{8}\left(1+\frac{\varkappa}{(1+\varkappa+v)}+\frac{v}{(1+\varkappa+v)}\right)$ for $(\theta, \varkappa, v) \in\left[0, \frac{1}{2}\right] \times \mathbb{R}^{2}$. Clearly, the function $f(0, \varkappa(0), v(0))=0$. Let $\varkappa, \varkappa^{*}, v, v^{*} \in \mathbb{R}$ and $\theta \in\left[0, \frac{1}{2}\right]$. Then we have

$$
\begin{aligned}
|f(\theta, \varkappa, v)| & \leq \frac{\theta^{2}}{8}\left(1+\frac{|\varkappa|}{(1+|\varkappa|+|v|)}+\frac{|v|}{(1+|\varkappa|+|v|)}\right) \\
& \leq \frac{1}{8}(1+|\varkappa|+|v|) .
\end{aligned}
$$

Hence condition $\left(H_{2}\right)$ is satisfied with $\kappa=\frac{1}{8}$. By choosing $\mathfrak{N}\left(\frac{1}{3}\right)=1$, we can find that $\eta_{1} \approx 0.23<1$. It follows from Theorem 3.2 that ABC-type nonlocal problem (4.3)-(4.4) has a solution on $\left[0, \frac{1}{2}\right]$.

Example 4.2 Consider the ABC-type nonlocal problem

$$
A B C \mathbb{D}_{0^{+}}^{\frac{1}{2}} \varkappa(\theta)=\frac{\theta}{\left(8+e^{\theta}\right)\left(1+|\varkappa(\theta)|+\left|{ }^{A B C} \mathbb{D}_{0^{+}}^{\frac{1}{2}} \varkappa(\theta)\right|\right)}, \quad \theta \in[0,1]
$$

with nonlocal conditions

$$
\frac{1}{4} \varkappa\left(\frac{1}{3}\right)+\frac{3}{4} \varkappa\left(\frac{1}{2}\right)=\varkappa_{0} \in \mathbb{R}
$$

where $0<\left(\tau_{1}=\frac{1}{3}, \tau_{2}=\frac{1}{2}\right)<1,\left(\beta_{1}=\frac{1}{4}, \beta_{2}=\frac{3}{4}\right)>0(k=1,2 ; m=2)$, and $\varrho=\frac{1}{2}$.

Notice that (4.3)-(4.4) is a particular case of (1.1) $-(1.2)$.

Set $f(\theta, \varkappa, v)=\frac{\theta}{8+e^{\theta}} \frac{1}{(1+\varkappa+v)}$ for $(\theta, \varkappa, v) \in[0,1] \times \mathbb{R}^{2}$. Clearly, the function $f(0, \varkappa(0)$, $v(0))=0$. Let $\varkappa, \varkappa^{*}, v, v^{*} \in \mathbb{R}$ and $\theta \in[0,1]$. Then we have

$$
\begin{aligned}
\left|f(\theta, \varkappa, v)-f\left(\theta, \varkappa^{*}, v^{*}\right)\right| & =\frac{\theta}{8+e^{\theta}}\left|\frac{1}{(1+\varkappa+v)}-\frac{1}{\left(1+\varkappa^{*}+v^{*}\right)}\right| \\
& \leq \frac{\theta}{8+e^{\theta}}\left(\frac{\left|\varkappa-\varkappa^{*}\right|+\left|v-v^{*}\right|}{(1+\varkappa+v)\left(1+\varkappa^{*}+v^{*}\right)}\right) \\
& \leq \frac{1}{8}\left[\left|\varkappa-\varkappa^{*}\right|+\left|v-v^{*}\right|\right] .
\end{aligned}
$$

Hence condition $\left(H_{1}\right)$ is satisfied with $L_{1}=\frac{1}{8}$. By choosing $\mathfrak{N}\left(\frac{1}{2}\right)=1$, we can find that $\Upsilon \approx 0.28$. Also, we have $\frac{1}{\mathcal{A}}=1 \neq 0$. It follows from Theorem 3.3 that problem (4.3)-(4.4) has a unique solution on $[0,1]$.

By Theorems 3.5 and 3.7, the solution of problem (4.3)-(4.4) depends continuously on the coefficients $\varkappa_{0}$ and $\sum_{k=1}^{m} \beta_{k}$. 


\section{Concluding remarks}

We can conclude that the main outcomes of this manuscript have been effectively accomplished. The existence and uniqueness of solutions for the nonlocal Cauchy problem for a nonlinear implicit $\mathrm{FDE}$ involving the $\mathrm{ABC}$ fractional derivative have been proved through some fixed point techniques (Theorems 2.8, 2.7) and some outcomes related to AB operators. Then, as an application, the continuous dependence of solution to such equations on arbitrary data involved therein was discussed. This paper adds and contributes to growth FDEs, particularly in the case of nonlocal implicit FDEs involving a novel fractional derivative presented recently by Atangana and Baleanu [11]. There are some works that carried out reported studies on the existence and continuous dependence of solutions of classical FDEs, and one of the destinations of this paper is to contribute with the goal that it can have a more prominent degree of studies identified with FDEs involving generalized fractional operators.

As a future direction, the studied problem would be interesting if it were studied on generalized fractional operators of variable order recently introduced by Yang and Machado [8] and its generalization by Sousa and Oliveira [47].

\section{Acknowledgements}

The authors extend their appreciation to the Deanship of Scientific Research at Imam Mohammad Ibn Saud Islamic University for funding this work through Research group no: RG-21-09-07. Also, the authors are grateful to the anonymous referees for the suggestions that improved the manuscript.

\section{Funding}

This research was supported by the Deanship of Scientific Research at Imam Mohammad Ibn Saud Islamic University, Grant No. RG-21-09-07.

Availability of data and materials

Not applicable.

\section{Declarations}

Competing interests

The authors declare that they have no competing interests.

\section{Authors' contributions}

The authors declare that the work was realized with equal contribution. All authors read and approved the final manuscript.

\section{Author details}

'Department of Mathematics and Statistics, Imam Mohammad Ibn Saud Islamic University, Riyadh, Saudi Arabia.

${ }^{2}$ Department of Mathematics, Hodeidah University, Al-Hudaydah, Yemen. ${ }^{3}$ Department of Basic Engineering Sciences, College of Engineering, Imam Abdulrahman Bin Faisal University, P.O. Box 1982, Dammam 34151, Saudi Arabia.

\section{Publisher's Note}

Springer Nature remains neutral with regard to jurisdictional claims in published maps and institutional affiliations.

Received: 10 June 2021 Accepted: 27 November 2021 Published online: 17 December 2021

\section{References}

1. Kilbas, A.A., Srivastava, H.M., Trujillo, J..: Theory and Applications of Fractional Differential Equations. North-Holland Math. Stud, vol. 204. Elsevier, Amsterdam (2006)

2. Podlubny, I.: Fractional Differential Equations. Academic Press, San Diego (1999)

3. Almeida, R.: A Caputo fractional derivative of a function with respect to another function. Commun. Nonlinear Sci. Numer. Simul. 44, 460-481 (2017)

4. Baleanu, D., Diethelm, K., Scalas, E., Trujillo, J.J.: Fractional Calculus Models and Numerical Methods. World Scientific, New York (2012)

5. Gambo, Y.Y., Jarad, F., Baleanu, D., Abdeljawad, T.: On Caputo modification of the Hadamard fractional derivatives. Adv. Differ. Equ. 2014, 10 (2014)

6. Sousa, J.V., de Oliveira, E.C.: On the $\psi$-Hilfer fractional derivative. Commun. Nonlinear Sci. Numer. Simul. 60, 72-91 (2018) 
7. Katugampola, U.: A new approach to a generalized fractional integral. Appl. Math. Comput. 218, 860-865 (2011)

8. Yang, X.J., Machado, J.T.: A new fractional operator of variable order: application in the description of anomalous diffusion. Phys. A, Stat. Mech. Appl. 481, 276-283 (2017)

9. Caputo, M., Fabrizio, M.: A new definition of fractional derivative without singular kernel. Prog. Fract. Differ. Appl. 1(2), 73-85 (2015)

10. Losada, J., Nieto, J.J.: Properties of a new fractional derivative without singular kernel. Prog. Fract. Differ. Appl. 1(2), 87-92 (2015)

11. Atangana, A., Baleanu, D.: New fractional derivative with non-local and non-singular kernel. Therm. Sci. 20(2), 757-763 (2016)

12. Abdeljawad, T., Baleanu, D.: Integration by parts and its applications of a new nonlocal fractional derivative with Mittag-Leffler nonsingular kernel. J. Nonlinear Sci. Appl. 9, 1098-1107 (2017)

13. Abdeljawad, T., Baleanu, D.: On fractional derivatives with exponential kernel and their discrete versions. Rep. Math. Phys. 80(1), 11-27 (2017)

14. Jarad, F., Abdeljawad, T., Hammouch, Z:: On a class of ordinary differential equations in the frame of Atangana-Baleanu fractional derivative. Chaos Solitons Fractals 117, 16-20 (2018)

15. Abdeljawad, T.: A Lyapunov type inequality for fractional operators with nonsingular Mittag-Leffler kernel. J. Inequal. Appl. 2017(1), 1 (2017)

16. Abdo, M.S., Panchal, S.K., Shah, K., Abdeljawad, T.: Existence theory and numerical analysis of three species prey-predator model under Mittag-Leffler power law. Adv. Differ. Equ. 2020(1), 249 (2020). https://doi.org/10.1186/s13662-020-02709-7

17. Abdo, M.S., Shah, K., Wahash, H.A., Panchal, S.K.: On a comprehensive model of the novel coronavirus (COVID-19) under Mittag-Leffler derivative. Chaos Solitons Fractals 135, 109867 (2020). https://doi.org/10.1016/i.chaos.2020.109867

18. Abdo, M.S., Abdeljawad, T., Ali, S.M., Shah, K.: On fractional boundary value problems involving fractional derivatives with Mittag-Leffler kernel and nonlinear integral conditions. Adv. Differ. Equ. 2021, 37 (2021). https://doi.org/10.1186/s13662-020-03196-6

19. Abdo, M.S., Abdeljawad, T., Kucche, K.D., Alqudah, M.A., Ali, S.M., Jeelani, M.B.: On nonlinear pantograph fractional differential equations with Atangana-Baleanu-Caputo derivative. Adv. Differ. Equ. 2021, 65 (2021). https://doi.org/10.1186/s13662-021-03229-8

20. Abdo, M.S., Abdeljawad, T., Shah, K., Jarad, F.: Study of impulsive problems under Mittag-Leffler power law. Heliyon 6(10), e05109 (2020). https://doi.org/10.1016/j.heliyon.2020.e05109

21. Algahtani, O.J.J.: Comparing the Atangana-Baleanu and Caputo-Fabrizio derivative with fractional order: Allen Cahn model. Chaos Solitons Fractals 89, 552-559 (2016)

22. Atangana, A., Koca, I.: Chaos in a simple nonlinear system with Atangana-Baleanu derivatives with fractional order. Chaos Solitons Fractals 89, 447-454 (2016)

23. Jajarmi, A., Baleanu, D.: A new fractional analysis on the interaction of HIV with CD4+ T-cells. Chaos Solitons Fractals $113,221-229(2018)$

24. Shabbir, S., Shah, K., Abdeljawad, T.: Stability analysis for a class of implicit fractional differential equations involving Atangana-Baleanu fractional derivative. Adv. Differ. Equ. 2021, 395 (2021). https://doi.org/10.1186/s13662-021-03551-1

25. Khan, H., Gomez-Aguilar, J.F., Abdeljawad, T., Khan, A.: Existence results and stability criteria for ABC-fuzzy-Volterra integro-differential equation. Fractals 28(08), 2040048 (2020)

26. Khan, A., Khan, H., Gomez-Aguilar, J.F., Abdeljawad, T.: Existence and Hyers-Ulam stability for a nonlinear singula fractional differential equations with Mittag-Leffler kernel. Chaos Solitons Fractals 127, 422-427 (2019)

27. Abdeljawad, T., Baleanu, D.: On fractional derivatives with generalized Mittag-Leffler kernels. Adv. Differ. Equ. 2018(1), $468(2018)$

28. Abdo, M.S., Abdeljawad, T., Shah, K., Ali, S.M.: On nonlinear coupled evolution system with nonlocal subsidiary conditions under fractal-fractional order derivative. Math. Methods Appl. Sci. 44(8), 6581-6600 (2021). https://doi.org/10.1002/mma.7210

29. Almeida, R., Malinowska, A.B., Monteiro, M.T.: Fractional differential equations with a Caputo derivative with respect to a kernel function and their applications. Math. Methods Appl. Sci. 41(1), 336-352 (2018)

30. Benchohra, M., Berhoun, F.: Impulsive fractional differential equations with variable times. Comput. Math. Appl. 59 1245-1252 (2010)

31. Gu, H., Trujillo, J.J.: Existence of mild solution for evolution equation with Hilfer fractional derivative. Appl. Math. Comput. 257, 344-354 (2015)

32. Benchohra, M., Bouriah, S., Nieto, J.J.: Terminal value problem for differential equations with Hilfer-Katugampola fractional derivative. Symmetry 11(5), 672 (2019)

33. Mei, Z.D., Peng, J.G., Gao, J.H.: Existence and uniqueness of solutions for nonlinear general fractional differential equations in Banach spaces. Indag. Math. 26, 669-678 (2015)

34. Benchohra, M., Henderson, J., Ntouyas, S.K., Ouahab, A.: Existence results for functional differential equations of fractional order. J. Math. Anal. Appl. 338, 1340-1350 (2008)

35. Wahash, H.A., Abdo, M.S., Panchal, S.K.: Fractional integrodifferential equations with nonlocal conditions and generalized Hilfer fractional derivative. Ufa Math. J. 11(4), 151-171 (2019)

36. Abdo, M.S., Panchal, S.K.: Some new uniqueness results of solutions to nonlinear fractional integro-differentia equations. Ann. Pure Appl. Math. 16, 345-352 (2018)

37. Abdo, M.S., Panchal, S.K.: Weighted fractional neutral functional differential equations. J. Sib. Fed. Univ. Math. Phys. 11 535-549 (2018)

38. Cao, J., Chen, H., Yang, W.: Existence and continuous dependence of mild solutions for fractional neutral abstract evolution equations. Adv. Differ. Equ. 2015(1), 1 (2015)

39. Haoues, M., Ardjouni, A., Djoudi, A.: Existence, interval of existence and uniqueness of solutions for nonlinear implicit Caputo fractional differential equations. TJMM 10(1), 9-13 (2018)

40. Hamd-Allah, E.M.A.: On the existence of solutions of two differential equations with a nonlocal condition. J. Egypt. Math. Soc. 24(3), 367-372 (2016) 
41. Kucche, K.D., Nieto, J.J., Venktesh, V.: Theory of nonlinear implicit fractional differential equations. Differ. Equ. Dyn. Syst. $28,1-17(2016)$

42. El-Sayed, A.M.A., Bin-Taher, E.O.: A nonlocal problem for a multiterm fractional-order differential equation. Int. J. Math. Anal. 5(29), 1445-1451 (2011)

43. El-Sayed, A.M.A., Gaafar, F., El-Gendy, M.: Continuous dependence of the solution of random fractional-order differential equation with nonlocal conditions. Fract. Differ. Calc. 7(1), 135-149 (2017)

44. El-Sayed, A.M.A., Alrashdi, M.A.: On the continuous dependence of a functional integral equation with parameter. Int. J. Math. Trends Technol. 65(3), 183-189 (2019)

45. Abdeljawad, T., Baleanu, D.: Discrete fractional differences with nonsingular discrete Mittag-Leffler kernels. Adv. Differ. Equ. 2016, 232 (2016). https://doi.org/10.1186/s13662-016-0949-5

46. Deimling, K.: Nonlinear Functional Analysis. Springer, Berlin (1985)

47. Sousa, J.V., de Oliveira, E.C.: Two new fractional derivatives of variable order with non-singular kernel and fractional differential equation. Comput. Appl. Math. 37(4), 5375-5394 (2018)

Submit your manuscript to a SpringerOpen ${ }^{\circ}$ journal and benefit from:

- Convenient online submission

- Rigorous peer review

- Open access: articles freely available online

- High visibility within the field

- Retaining the copyright to your article

Submit your next manuscript at $\gg$ springeropen.com 